\title{
Mandatory COVID vaccines and Fermi's paradox
}

\author{
Gilbert Berdine MD
}

"It's the end of the world as we know it. It's the end of the world as we know it. It's the end of the world as we know it, and I feel fine."

R.E.M. It's The End Of The World

What is Fermi's Paradox? What could COVID vaccines have anything to do with Fermi's Paradox? The answer to these questions should give pause to anyone advocating for mandatory COVID vaccination.

Fermi's Paradox has to do with the likelihood that life exists on other planets. ${ }^{1}$ In other words, we are not alone. Fermi was not even close to the first one to ponder this question; the question has been debated ever since man was aware that lights in the night sky represented stars and that these stars might have planets orbiting around them just as the Earth orbits its star-the Sun. Fermi's name is attached to the paradox because of the answer he gave to the question: "But where is everybody?"

Fermi's reasoning was as follows: suppose that life on planet Earth is not unique; there are many stars with many planets; there are so many planets that there should be many examples with life even if life is a rare event; there is nothing special about the Sun and Earth, so many of these examples should be older with more advanced civilizations; there should be so many advanced civilizations who have found Earth and humans that we should not be able to avoid them on a daily basis; we should be either a conquest or a trade hub. Fermi's answer, "But where is everybody?" became famous, in part, because it was part of a discussion in a cafeteria at Los Alamos during the Manhattan project, and his audience included Edward Teller, the inventor of the hydrogen bomb.

Corresponding author: Gilbert Berdine Contact Information: Gilbert.Berdine@ttuhsc.edu DOI: 10.12746/swrccc.v9i39.833
There are several classes of answers to Fermi's Paradox. The simplest class of answer is that life on Earth is unique, so there is no reason to find life elsewhere. The other classes of answers assume that life on Earth was not unique, that there are many examples of life on other planets, but that there are good reasons that we have not come in contact. The class of answers relevant to this discussion has to do with extinction. Contact requires the ability to travel between stars or between galaxies, and the natural progress of knowledge may lead first to other technologies that cause the extinction of life. The usual examples given are destruction by thermonuclear war or degradation of the planet through pollution, overpopulation, or excessive heat production. This theme has been explored by science fiction. Many zombie apocalypse scenarios are triggered by the unintended consequences of human engineered viruses, or other catastrophic consequences of tampering with viruses. Rise of the Planet of the Apes is based on a viral drug intended to cure dementia that ends up making apes intelligent and killing humans. A recent television series, Utopia, was produced and released by Amazon Prime Video. The plot involves a billionaire oligarch who fakes a viral outbreak, claims the virus is an existential threat, and conveniently has a vaccine, the true and nefarious purpose of which is to sterilize a substantial portion of humanity to save us from overpopulation. Amazon has claimed that any resemblances in the plot to real events are coincidence.

Which brings us to mandatory COVID-19 vaccinations. The development of COVID vaccines has been rushed. U.S. government agencies have been quick to assure the public that the vaccines are efficacious and safe, but there are insufficient data to make such 
claims. The clinical trials used for FDA approval had around 10,000 subjects in both the vaccine arm and control arm, so harmful effects less common than $1 / 10,000$ could not possibly be detected. As for efficacy, no COVID related deaths were seen in either the vaccine arm or the control arm, so this study had no evidence whatsoever that vaccination prevented death from COVID-19. ${ }^{2}$ A larger trial of the vaccine in Israel found that over 26,000 vaccinations were necessary to prevent a single death from COVID-19. ${ }^{3}$ Of course, the data were not presented that way; rather a high efficacy figure was quoted based on the reduced odds of converting a PCR test to positive from negative despite the fact that the number to vaccinate in order to prevent a single PCR test conversion was $364 .^{3}$

There are data for efficacy of the vaccine, but the benefit is small. COVID-19 does not represent an existential threat to humanity. The benefit of the vaccine is not large enough to justify mandatory vaccination, yet governments are already considering mandatory or coerced vaccination programs. Boris Johnson is facing a backlash after suggesting that Brits be required to hold a "vaccine passport" in order to order a pint in a British pub. ${ }^{4}$ Dr. Anthony Fauci said, "Everything will be on the table for discussion" in answer to questions about COVID-19 "vaccine passports" and mandatory vaccinations in the United States. ${ }^{5}$ President Joe Biden wants "vaccine passports" to permit dining out in the United States. ${ }^{6}$ This is rather remarkable considering we have less than a year of data regarding vaccine safety.

It has been argued that the vaccine could pose a threat to human fertility. The so-called fact checkers claim this concern has been debunked. ${ }^{7}$ However, the claims debunked do not precisely match the claims made by the concerned. This is called knocking down a straw man. The precise theoretical concern was that the spike protein coded for by the messenger RNA of the vaccine shares an amino acid sequence with the syncytin-1 protein that is part of human placenta. The theoretical concern was that the vaccine could induce an antibody response that might cross react to human placenta and could cause miscarriages in pregnant women. The debunkers point out that the spike protein is not an exact match to syncytin-1; this is a straw man as nobody ever claimed an exact match. The debunkers argue that there is no evidence that COVID-19 disease causes antibodies against syncytin-1, but absence of evidence is not evidence of absence. The debunkers also claim that if the concern was valid, there should be evidence of reduced fertility following COVID-19 infection and no such evidence exists. However, evidence of reduced fertility 9 months after the pandemic exists is popping up everywhere; it is unclear whether the reduced fertility is due to adverse effects of lockdowns on behavior or whether the reduced fertility has a biologic basis. Whether this particular claim about risk to fertility has any merit or not is not the point. The point is why would governments force people to take a vaccine that might put fertility at risk when the benefit is very small and the virus being vaccinated against does not pose an existential threat? If the vaccine represents even a theoretically possible existential threat, it is better to be safe than sorry.

The COVID-19 vaccine was discussed in detail in the previous issue of the Journal. ${ }^{8}$ Let the people who volunteer to be vaccinated get the vaccine. Let the people who are skeptics decline the vaccine. Humanity is better off with a significant cohort of unvaccinated people to permit long term study of efficacy and safety of the vaccine. If vaccines are mandatory, we will never know whether the vaccines were efficacious or safe. If the safety issue turns out to be an existential threat, it will be too late to prevent humans from becoming an example of Fermi's Paradox.

Keywords: COVID-19, Vaccination, Fermi's paradox, safety, efficacy

Article citation: Berdine G. Mandatory COVID vaccines and Fermi's paradox. The Southwest Respiratory and Critical Care Chronicles 2021;9(39):76-78

From: Department of Internal Medicine, Texas Tech University Health Sciences Center, Lubbock, Texas

Submitted: $3 / 30 / 2021$

Accepted: 4/2/2021

Conflicts of interest: none

This work is licensed under a Creative Commons Attribution-ShareAlike 4.0 International License. 


\section{REFERENCES}

1. Fermi paradox.Wikipedia.https://en.wikipedia.org/wiki/Fermi_ paradox. Accessed 3/30/2021.

2. Berdine $\mathrm{G}$. How much does the Pfizer vaccine actually reduce risk of hospitalization? Mises Institute. https://mises.org/ wire/how-much-does-pfizer-vaccine-actually-reduce-riskhospitalization. Accessed 3/30/2021.

3. Berdine $\mathrm{G}$. What we've learned from Israel's Covid vaccine program. Mises Institute. https://mises.org/wire/what-wevelearned-israels-covid-vaccine-program. Accessed 3/30/2021.

4. Craig J. COVID-19: Publicans and Tory MPs' fury at PM's 'vaccine passports' for pubs idea. Sky News. https://news. sky.com/story/covid-19-publicans-and-tory-mps-fury-at-pmsvaccine-passports-for-pubs-idea-12255917. Accessed 3/30/2021.
5. Kim S. Dr. Fauci on mandatory COVID vaccines: 'Everything will be on the table.' Newsweek. https://www.newsweek.com/ coronavirus-anthony-fauci-covid-vaccine-passport-mandatoryvaccinations-travel-1558303. Accessed 3/30/2021.

6. Diamond D, Sun LH, Stanley-Becker I. 'Vaccine passports' are on the way, but developing them won't be easy. The Washington Post. https://www.washingtonpost.com/health/2021/03/28/ vaccine-passports-for-work/. Accessed 3/30/2021.

7. Goodman B. Why COVID vaccines are falsely linked to infertility. WebMD. https://www.webmd.com/vaccines/covid19-vaccine/news/20210112/why-covid-vaccines-are-falselylinked-to-infertility. Accessed 3/30/2021.

8. Armin S, Wakil A, Tarbox J, et al. COVID-19 vaccination: an attempt to control the pandemic. The Southwest Respiratory and Critical Care Chronicles 2021;9(37):32-39.

\section{Addendum:}

Please see a second commentary by James A Tarbox in this issue of the journal. 